\title{
Pathogenic role of mast cells in the development of diabetic nephropathy: a study of patients at different stages of the disease
}

\author{
J. M. Zheng • G. H. Yao • Z. Cheng • R. Wang • \\ Z. H. Liu
}

Received: 3 August 2011 / Accepted: 4 November 2011 /Published online: 1 December 2011

(C) Springer-Verlag 2011

\begin{abstract}
Aims/hypothesis Increased renal mast cells have been detected in diabetic nephropathy. However, only a few patients have been examined. Evidence of the involvement of mast cells in diabetic nephropathy is still scarce, and no observation of mast cells during the development of diabetic nephropathy has yet been reported in humans. Here, we examined changes in renal mast cells in patients at different stages of diabetic nephropathy and related these to the development of the disease.

Methods Eighty patients at different clinical stages of diabetic nephropathy and 16 normal kidney donors were recruited. Immunohistochemical staining for tryptase, chymase, TGF- $\beta 1$, renin and TNF- $\alpha$ was done on renal sections from patients and control participants. Changes in mast cell number, degranulation, subtype and phenotype were examined. Correlation between mast cells and patients' clinical and pathological indices was analysed.

Results With progression of diabetic nephropathy, the number and degranulation level of mast cells increased. Increase in mast cell number and degranulation level correlated significantly with tubular interstitial injury. Almost all renal mast cells in patients with diabetic nephropathy were found to produce chymase, renin, TGF$\beta 1$ and TNF- $\alpha$. The level of TNF- $\alpha$ in mast cells increased with progression of diabetic nephropathy.

Conclusions/interpretation This study suggests that mast cells are involved in development of diabetic nephropathy. Through release of bioactive substances, such as tryptase,
\end{abstract}

J. M. Zheng $\cdot$ G. H. Yao $\cdot$ Z. Cheng $\cdot$ R. Wang $\cdot$ Z. H. Liu $(\varangle)$

Research Institute of Nephrology, Jingling Hospital,

Nanjing University School of Medicine,

305 East Zhongshan Road,

Nanjing 210002, People's Republic of China

e-mail: zhihong-liu@hotmail.com chymase, TGF- $\beta 1$, renin and TNF- $\alpha$, into the tubular interstitium by degranulation, mast cells could promote renal inflammation and fibrosis, and thus contribute to diabetic nephropathy.

Keywords Chymase - Diabetic nephropathy - Fibrosis . Inflammation · Mast cells $\cdot$ Renin $\cdot$ TGF- $\beta 1 \cdot$ TNF- $\alpha$.

Tryptase

$\begin{array}{ll}\text { Abbreviations } \\ \text { eGFR } & \text { Estimated glomerular filtration rate } \\ \text { MC } & \text { Mast cell } \\ \text { NAG } & N \text {-acetyl- } \beta \text {-glucosaminidase } \\ \text { RBP } & \text { Retinol-binding protein } \\ \text { RIV } & \text { Relative interstitial volume } \\ \text { UAE } & \text { Urinary albumin excretion rate }\end{array}$

\section{Introduction}

Diabetic nephropathy is a multistage clinical syndrome characterised by thickening of the glomerular basement membrane and mesangial expansion with progression into glomerulosclerosis, tubular necrosis and interstitial fibrosis, which ultimately result in renal failure. Although a lot of investigations have been carried out, the pathogenic mechanism involved in diabetic nephropathy is not completely understood and no satisfactory therapeutic option is available to treat this insidious disease [1].

Mast cells (MCs) are a group of multifunctional cells. Knowledge obtained in the past two decades indicates that MCs are not only the key effector cells in anaphylaxis, but also regulators of innate and adaptive immune responses [2, 3]. The pathogenic roles of MCs have been extended to 
allergic diseases and helminth infection, and other diseases such as autoimmune diseases, inflammatory diseases, carcinogenesis and fibrosis [3-10]. With regards to the production of tryptase and chymase, two subtypes of MCs have been reported in humans: the $\mathrm{MC}_{\mathrm{T}}$ subtype is believed to produce tryptase only, whereas the $\mathrm{MC}_{\mathrm{TC}}$ subtype is believed to produce both tryptase and chymase.

MCs have been detected in a lot of renal diseases [11-22]; however, only a few cases were investigated in each kind of renal disease in most of the studies reported. Observation of MCs in the development of renal disease is still scarce. Evidence provided by previous studies suggests that MCs contribute to renal diseases mainly by promoting tubular interstitial injury; however, their exact role remains poorly understood. Increased numbers of MCs have been reported in diabetic nephropathy $[11,13$, 17], but, again, only a few patients were examined. Evidence of the involvement of MCs in diabetic nephropathy is still scarce, and no observation has been made of MCs during the development of diabetic nephropathy in humans. The aim of the present study was to examine changes in MC number, degranulation level, subtype and phenotype in the renal tissues of patients at different stages of diabetic nephropathy and to relate this to the development of disease.

\section{Methods}

Study population Eighty type 2 diabetic patients with biopsy-proven diabetic nephropathy, who were hospitalised at the clinical unit of the nephrology centre of Jingling Hospital from 2003 to 2008, were recruited. All these patients met the World Health Organization criteria for type 2 diabetes mellitus, and renal biopsy consistent with the diagnosis of diabetic nephropathy and exclusion for other concomitant renal diseases. According to their clinical characteristics (regardless of duration of diabetes), patients were classified into microalbuminuria (urinary albumin excretion rate [UAE] $>30 \mathrm{mg} / 24 \mathrm{~h}$ and $<300 \mathrm{mg} / 24 \mathrm{~h}$, $n=19$ ), proteinuria (UAE $\geq 300 \mathrm{mg} / 24 \mathrm{~h}$ and estimated glomerular filtration rate [eGFR, according to the equation of Modification of Diet in Renal Disease] $\geq 60 \mathrm{ml} \mathrm{min}^{-1}$ $1.73 \mathrm{~m}^{-2}, n=32$ ) and renal insufficiency (eGFR $\left.<60 \mathrm{ml} \mathrm{min}{ }^{-1} 1.73 \mathrm{~m}^{-2}, n=29\right)$ stage groups. Patients with hypertension were given angiotensin-receptor blockers such as irbesartan or valsartan. Other antihypertensive agents (calcium-channel blockers, ACE inhibitors, diuretics, etc.) were also used as needed. The target blood pressure was the same (a systolic blood pressure of $135 \mathrm{mmHg}$ or less, and a diastolic blood pressure of $85 \mathrm{mmHg}$ or less). Sixteen normal renal tissues from mismatched kidney donors served as normal control participants (normal control group). Informed consent was obtained from participants. All research work with human participants was in accordance with the ethical standards of the responsible committee on human experimentation and with the Declaration of Helsinki. Table 1 shows relevant clinical and pathological characteristics of the patients.

Histology For light microscopy, renal biopsy specimens were fixed in $10 \%$ neutral buffered formalin, embedded in paraffin and sectioned at $2 \mu \mathrm{m}$ thickness. Haematoxylineosin, periodic acid-Schiff's reagent, Masson's trichrome and periodic acid methenamine silver staining were carried out. Glomerular, tubular interstitial and vascular lesions in biopsies were recorded, classified and scored according to criteria described by Tervaert et al. [23]. Immunofluorescence included the use of antibodies against $\operatorname{IgA}, \operatorname{IgG}, \operatorname{IgM}$, $\mathrm{C} 3, \mathrm{C} 1 \mathrm{q}$, and $\mathrm{k}$ and $\lambda$ light chains to rule out other renal diseases. Electron microscopy was also performed to confirm the diagnosis.

Neutrophils, eosinophils and plasma cells were identified according to the morphological characteristics of these cells on haematoxylin and eosin-stained sections, and the number of these cells in the tubular interstitium was counted by two pathologists. Immunohistochemical staining for CD3, CD20 and CD68 was performed to label $\mathrm{T}$ lymphocytes, $\mathrm{B}$ lymphocytes and macrophages respectively. The numbers of $\mathrm{CD} 3, \mathrm{CD} 20$ and CD68 positive cells in the tubular interstitium were counted. For each section, at least ten randomly selected cortical regions were examined, and the area of the selected regions in each section was measured using NIS Element BR3.4 software (Nikon, Shinagaw-ku, Tokyo, Japan). The number of each kind of inflammatory cell (including neutrophils, eosinophils, plasma cells, T lymphocytes, B lymphocytes and macrophages) was presented as the number of the cells per $\mathrm{mm}^{2}$ of renal cortex area.

The relative interstitial volume (RIV) was measured according to methods described by Okon et al. [17, 24]. For each section, ten randomly selected cortical regions were measured and the average value was taken as the RIV of the section.

Evaluation of the degranulation level of MCs Renal sections were stained with Toluidine Blue and examined under a high power field by two different researchers using a single blind approach. Degranulated MCs were determined by the presence of extruded granule contents [25]. At least 20 randomly selected fields were examined for each section. The total numbers of Toluidine Blue staining-positive cells (total MCs) and of degranulated MCs were counted. The degranulation level of MCs in each section was presented as the ratio of degranulated MCs to total MCs. 
Table 1 Clinical and pathological variables of participating patients

\begin{tabular}{|c|c|c|c|}
\hline Variable & $\begin{array}{l}\text { Microalbuminuria } \\
\text { stage group }\end{array}$ & $\begin{array}{l}\text { Proteinuria stage } \\
\text { group }\end{array}$ & $\begin{array}{l}\text { Renal insufficiency } \\
\text { stage group }\end{array}$ \\
\hline$n$ & 19 & 32 & 29 \\
\hline Age (years) & $46(43-48)$ & $49(45-57)$ & $53(46-57)$ \\
\hline Duration of diabetes mellitus (months) & $48(34-72)$ & $84(36.0-153.8)$ & $148(90-152)^{* * \dagger}$ \\
\hline Male/female & $11 / 8$ & $16 / 16$ & $16 / 13$ \\
\hline BMI $\left(\mathrm{kg} / \mathrm{m}^{2}\right)$ & $26.0(20.6-27.7)$ & $25.1(23.1-27.3)$ & $25.9(22.1-27.5)$ \\
\hline Fasting blood glucose (mmol/l) & $6.2(5.6-7.1)$ & $7.0(6.0-8.6)$ & $7.0(5.4-7.8)$ \\
\hline Postprandial blood glucose (mmol/l) & $10.0(8.4-13.0)$ & $10.9(9.2-13.5)$ & $11.9(11.0-13.1)$ \\
\hline Serum creatinine $(\mu \mathrm{mol} / \mathrm{l})$ & $63.6(54.8-77.8)$ & $69.0(56.4-89.9)$ & $142.3(125.1-202.4)^{* *+\dagger}$ \\
\hline Serum cystatin C (mg/l) & $0.9(0.8-1.0)$. & $1.1(1.0-1.3)$ & $2.0(1.6-2.6)^{* * \dagger \dagger}$ \\
\hline Serum albumin $(g / l)$ & $43.7(42.4-47.1)$ & $39.1(32.6-43.2)^{* *}$ & $34.5(30.1-38.4)^{* *}$ \\
\hline Haemoglobin $(g / l)$ & $142(128.5-152.5)$ & $128(109.0-141.5)^{* *}$ & $102(93.5-117.5)^{* * \dagger \dagger}$ \\
\hline Glycated haemoglobin (\%) & $6.8(6.0-7.5)$ & $7.0(6.4-8.5)$ & $6.6(6.3-7.4)$ \\
\hline Glycated haemoglobin (mmol/l) & $50.3(42.1-57.9)$ & $53.0(46.4-68.9)$ & $48.6(45.4-57.4)$ \\
\hline Serum cholesterol $(\mathrm{mmol} / \mathrm{l})$ & $4.0(3.8-5.3)$ & $5.2(4.0-6.7)$ & $5.0(4.2-6.3)$ \\
\hline Serum triacylglycerol (mmol/l) & $1.6(1.1-2.4)$ & $2.0(1.6-2.7)$ & $1.7(1.2-2.9)$ \\
\hline Urine C3 (mg/l) & $2.8(2.5-3.0)$ & $3.1(2.7-8.1)^{*}$ & $18.0(5.1-30.6)^{* * \dagger}$ \\
\hline Urine $\alpha 2-$ microglobulin $(\mathrm{mg} / \mathrm{l})$ & $2.9(2.5-3.5)$ & $3.0(2.8-5.4)$ & $4.1(3.0-7.5)^{* * \dagger \dagger}$ \\
\hline Urine lysozyme (mg/l) & $0.5(0.5-1.0)$ & $0.5(0.5-0.9)$ & $1.6(0.7-4.6)^{* * \dagger \dagger}$ \\
\hline Urine RBP (mg/l) & $0.3(0.1-0.4)$ & $0.5(0.2-1.6)$ & $3.6(2.0-7.1)^{* * \dagger \dagger}$ \\
\hline Urine NAG (U/mmol creatinine) & $1.3(0.8-1.9)$ & $3.1(1.7-5.8)^{* *}$ & $5.6(3.8-6.8)^{* * \dagger}$ \\
\hline Proteinuria $(g / 24 h)$ & $0.2(0.1-0.3)$ & $1.1(0.8-2.8)^{* *}$ & $2.6(1.9-4.0)^{* * \dagger}$ \\
\hline Urinary osmotic pressure (mosmol $/ \mathrm{kg} \mathrm{H}_{2} \mathrm{O}$ ) & $754(425-825)$ & $466.5(341.5-785.8)$ & $445(354.5-636.5)^{* *}$ \\
\hline eGFR $\left(\mathrm{ml} \mathrm{min} \min ^{-1} 1.73 \mathrm{~m}^{-2}\right)$ & $105.6(98.7-121.8)$ & $92.4(76.6-115.5)$ & $43.7(29.1-49.9)^{* *+\dagger}$ \\
\hline Systolic blood pressure (mmHg) & $120(110-130)$ & $140(124.8-150)$ & $130(122-147.5)$ \\
\hline Diastolic blood pressure (mmHg) & $80(71-80)$ & $85.5(75.8-90)$ & $84(80-90)$ \\
\hline Hypertension, $n(\%)$ & $8(42.1)$ & $25(78.1)$ & $24(82.8)$ \\
\hline \multicolumn{4}{|l|}{ Glomerular lesions, $n(\%)$} \\
\hline Class Ia & $19(100)$ & $8(25.0)$ & $0(0)$ \\
\hline Class IIb & $0(0)$ & $6(18.8)$ & $4(13.8)$ \\
\hline Class III & $0(0)$ & $18(56.3)$ & $18(62.1)$ \\
\hline Class IV & $0(0)$ & $0(0)$ & $7(24.1)$ \\
\hline \multicolumn{4}{|l|}{ Interstitial lesions (score), $n(\%)$} \\
\hline Score $=1$ & $4(21.1)$ & $0(0)$ & $0(0)$ \\
\hline Score $=2$ & $13(68.4)$ & $13(40.6)$ & $0(0)$ \\
\hline Score $=3$ & $2(10.5)$ & $16(50.0)$ & $6(20.7)$ \\
\hline Score $=4$ & $0(0)$ & $3(9.4)$ & $12(41.4)$ \\
\hline Score $=5$ & $0(0)$ & $0(0)$ & $11(37.9)$ \\
\hline \multicolumn{4}{|l|}{ Vascular lesions (score), $n(\%)$} \\
\hline Score $=0$ & $1(5.3)$ & $0(0)$ & $0(0)$ \\
\hline Score $=1$ & $6(31.6)$ & $1(3.1)$ & $0(0)$ \\
\hline Score $=2$ & $5(26.3)$ & $5(15.6)$ & $1(3.5)$ \\
\hline Score $=3$ & $7(36.8)$ & $17(53.1)$ & $15(51.7)$ \\
\hline Score $=4$ & $0(0)$ & $9(28.1)$ & $13(44.8)$ \\
\hline
\end{tabular}

Data are median (interquartile range), unless otherwise indicated

${ }^{*} p<0.05,{ }^{*} p<0.01$ vs microalbuminuria stage group; ${ }^{\dagger} p<0.05,{ }^{\dagger \dagger} p<0.01$ vs proteinuria stage group 
Immunohistochemistry Immunohistochemical staining was performed using routine methods. Briefly, formalin-fixed sections were deparaffinised with xylene and rehydrated with graded ethanols. The sections were autoclaved for antigen repair. Endogenous peroxidase was blocked with hydrogen peroxide. For reduction of background labelling, the sections were blocked for $30 \mathrm{~min}$ in $10 \%$ fetal calf serum. The samples were rinsed in PBS and then incubated overnight at $4^{\circ} \mathrm{C}$ with different first antibodies, such as mouse anti-human MC tryptase monoclonal antibody (Chemicon, Temicon, CA, USA, 1:2,000), mouse antihuman MC chymase monoclonal antibody (Chemicon, 1:1,000), rabbit anti-human renin polyclonal antibody (Santa Cruz Biotechnology, CA, USA, 1:200), mouse anti-human TNF- $\alpha$ monoclonal antibody (Santa Cruz Biotechnology, 1:100), rabbit anti-human TGF- $\beta 1$ polyclonal antibody (Santa Cruz Biotechnology, 1:200), mouse anti-human CD3 monoclonal antibody (Novocastra, UK, 1:200), mouse anti-human CD20 monoclonal antibody (Dako, Glostrup, Denmark, 1:100) and mouse anti-human CD68 monoclonal antibody (Dako, 1:100). After washing with PBS, the tissues were incubated with Envision reagent (Dako) for $45 \mathrm{~min}$, then the sections were washed and developed with diaminobenzidine. Each section was counterstained with haematoxylin. Normal homologous serum was used to replace the first antibody as a negative control.

Measurement of renal MCs Immunohistochemical staining for tryptase was carried out to mark all the renal MCs. The cortex part of each renal section was fully scanned and photographed under a light microscope by a CCD (Nikon DS-Ri1, Nikon), which was connected to a personal computer. Then, the number of tryptase-positive MCs in the cortex of each section was counted by two independent researchers using a single blind approach. The renal tissue area of the same region was measured using NIS Element BR software. The number of renal MCs in each section was presented as the number of MCs per $\mathrm{mm}^{2}$ of renal cortex area (MCs density).

Analysis of the subtype of MCs Immunohistochemical staining for tryptase and chymase was carried out on serial renal sections. The MCs that were positive for tryptase staining and negative for chymase staining were believed to be $\mathrm{MC}_{\mathrm{T}}$ subtype. The MCs that were positive for both tryptase and chymase staining were believed to be $\mathrm{MC}_{\mathrm{TC}}$ subtype. To measure the ratio of each subtype, at least ten randomly selected high power fields were examined. The number of each subtype of $\mathrm{MC}$ was counted and the ratio was calculated for each section.

Semi-quantitative analysis of TNF- $\alpha$ in renal MCs The TNF- $\alpha$ level in MCs was analysed quantitatively using
Image-pro Plus 6.0 software (Media Cybernetics, Bethesda, MD, USA). Serial renal sections were used in the assay. For each pair of serial sections, one section was stained for tryptase to mark MCs, and the other was stained for TNF$\alpha$. At least 15 randomly selected MCs were analysed from each section of the diabetic nephropathy patients. As only a few MCs were found in each section of the normal control participants, all MCs found in all sections were pooled together and analysed.

Statistical analysis All data were analysed using SPSS version 10.0 (SPSS, Chicago, IL, USA). Because the data involved a small number of participants (for each group) or were determined to be non-normal, all the data are presented as median (interquartile range), and multiple comparisons were performed using Kruskal-Wallis ANOVA, with pairwise comparison performed using Mann-Whitney $U$ test with Bonferroni correction. Correlation analysis was carried out using Spearman coefficients with Bonferroni correction to adjust for the multiple testing. $p<0.05$ was considered statistically significant.

\section{Results}

Distribution of MCs in renal tissues of patients with diabetic nephropathy MCs were detected in the cortex and medulla of renal sections from diabetic nephropathy patients by immunohistochemical staining for tryptase, which is the specific molecular marker of MCs. Most MCs were found to be distributed diffusely in the interstitium, especially in the peritubular, perivascular and periglomerular regions (Fig. 1b). Occasionally, the MCs were found to be infiltrated in the wall of atrophied tubules. Few intraglomerular MCs were detected in our tissue samples. MCs were mostly located on the periphery rather than within areas where inflammatory cells aggregated.
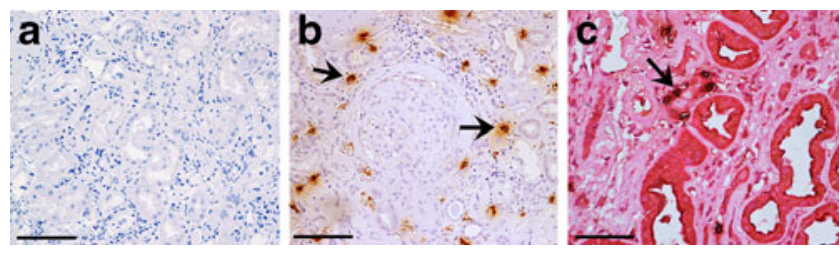

Fig. 1 Distribution of MCs in the renal tissues of patients with diabetic nephropathy. MCs (shown by black arrows) were found to be distributed diffusely in the interstitium. a Negative control of immunohistochemistry (original magnification $\times 200$ ). Scale bar, $100 \mu \mathrm{m}$. b Occasionally, the MCs were found to be infiltrated in the wall of atrophied tubules (original magnification $\times 200$ ). Scale bar, $100 \mu \mathrm{m}$. c Immunohistochemical staining for tryptase was used to mark the MCs. To show the renal structure better, haematoxylin-eosin staining was performed after immunohistochemical staining (original magnification $\times 400$ ). Scale bar, $50 \mu \mathrm{m}$ 
Renal MCs increased as the disease progressed In the normal control group, only a few MCs were found. The density of MCs in the normal control group was $2.2(0.3-$ 3.7) $\mathrm{MCs} / \mathrm{mm}^{2}$. However, increased numbers of MCs were found in the diabetic nephropathy patients and these paralleled the progression of the disease (Fig. 2).

Degranulation level of MCs increased with the development of diabetic nephropathy The degranulation level of MCs was analysed using Toluidine Blue staining. In the normal control group, degranulation was found in only a few MCs (about one in ten). However, with the development of diabetic nephropathy, the degranulation level of MCs increased significantly (Fig. 3).

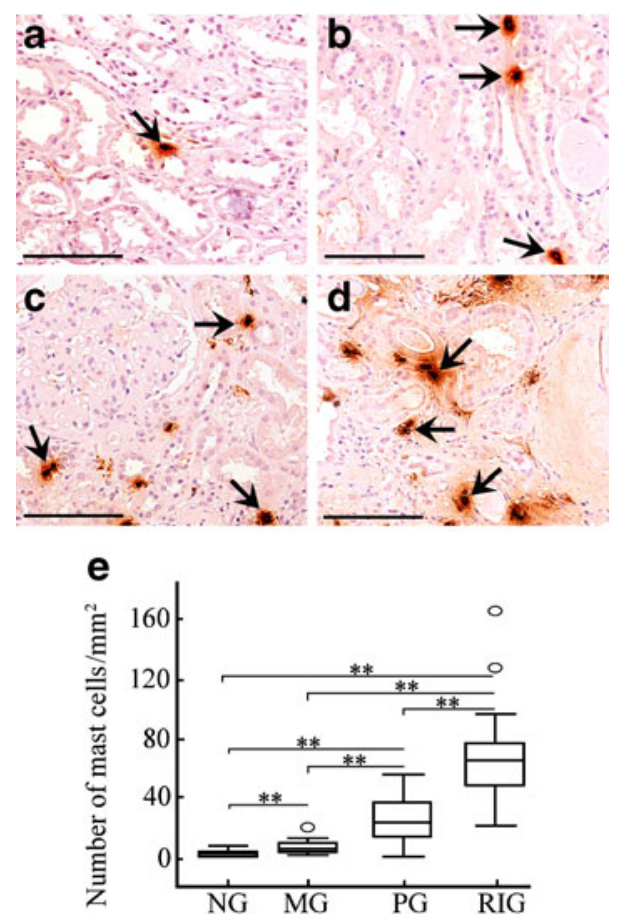

Fig. 2 Renal MCs (shown by black arrows) increased with development of diabetic nephropathy. Sections of renal biopsies from 80 patients with diabetic nephropathy (including the microalbuminuria stage group $[n=19]$, proteinuria stage group $[n=32]$ and renal insufficiency stage group $[n=29]$ ) and 16 normal control participants were used in the assay. Immunohistochemical staining for tryptase was carried out to mark renal MCs. The number of tryptase-positive cells in the cortex part of each section was counted. The area of the same part was measured and the density of MCs in renal cortex was calculated. Multiple comparisons were performed using KruskalWallis ANOVA, and pairwise comparison was performed using Mann-Whitney $U$ test, with Bonferroni correction. a Normal control group. b Microalbuminuria stage group. c Proteinuria stage group. d Renal insufficiency stage group. Scale bars for (a-d) $100 \mu \mathrm{m}$. e Results of statistical analysis, $* * p<0.01$. White circles represent extreme values where the extremes are defined as cases with values more than three box lengths from the upper or lower edge of the box. The box length is the interquartile range. MG, microalbuminuria stage group; NG, normal control group; PG, proteinuria stage group; RIG, renal insufficiency stage group

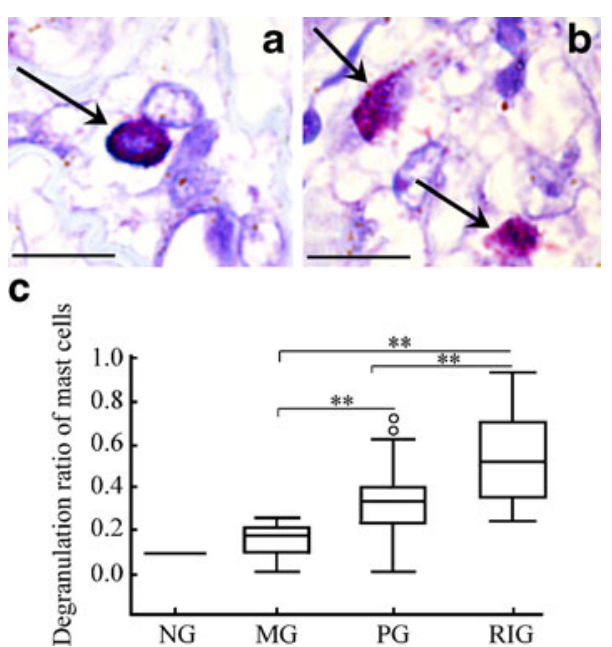

Fig. 3 Increased degranulation level of renal MCs (shown by black arrows) was found with development of diabetic nephropathy. Sections of renal biopsies from 80 patients with diabetic nephropathy (including microalbuminuria stage group $[n=19]$, proteinuria stage group [ $n=32$ ] and renal insufficiency stage group [ $n=29]$ ) and 16 normal control participants were used in the assay. For the diabetic nephropathy patients, at least 20 randomly selected high power fields were examined and the ratio of degranulated MCs was calculated for each section. As only a few MCs were found in each section from the normal controls, all MCs found in all sections from the normal controls were pooled together and the ratio of degranulated MCs was calculated (three degranulated MCs were found in $31 \mathrm{MCs}$ ). Multiple comparisons were performed using Kruskal-Wallis ANOVA, and pairwise comparison was performed using Mann-Whitney $U$ test, with Bonferroni correction. a MCs without degranulation (note that the MC is intact and no granule was found outside the cell). b Degranulated MCs (note that some granules have been released outside the cell). Scale bars for (a) and (b) $10 \mu \mathrm{m}$. c Results of statistical analysis: the horizontal line was represents the average degranulation level of the normal control group, ${ }^{* *} p<0.01$. White circles represent extreme values where the extremes are defined as cases with values more than three box lengths from the upper or lower edge of the box. The box length is the interquartile range. MG, microalbuminuria stage group; $\mathrm{NG}$, normal control group; $\mathrm{PG}$, proteinuria stage group; RIG, renal insufficiency stage group

Correlation of renal MCs with clinical and pathological indices As shown in Table 2, density of renal MCs was found to be correlated significantly with eGFR, serum creatinine, serum cystatin $\mathrm{C}$, urine retinol-binding protein (RBP), urine lysozyme, urine $N$-acetyl- $\beta$-glucosaminidase (NAG), urine $\mathrm{C} 3$, urine $\alpha 2$ microglobulin and $24 \mathrm{~h}$ urine protein level. Significant correlation was also found between the density of renal MCs and the RIV. Moreover, MCs were found to be significantly correlated with the number of renal tubular interstitial inflammatory cells, including neutrophils, eosinophils, macrophages and $\mathrm{T}$ lymphocytes. However, plasma cells and B lymphocytes were not found to be correlated with MCs.

Most MCs were found to be $M C_{T C}$ subtype and no change in $M C$ subtype was found during the development of 
Table 2 Correlation of renal MCs with clinical and pathological indices

\begin{tabular}{|c|c|c|c|}
\hline \multirow[t]{2}{*}{ Indices } & \multirow[t]{2}{*}{$n$} & \multicolumn{2}{|c|}{ Mast cell number $/ \mathrm{mm}^{2}$} \\
\hline & & $r$ & $p$ value \\
\hline \multicolumn{4}{|l|}{ Interstitial infiltrating cells } \\
\hline Interstitial neutrophils & 80 & 0.616 & $<0.0001$ \\
\hline Interstitial macrophages & 80 & 0.701 & $<0.0001$ \\
\hline Interstitial $\mathrm{T}$ lymphocytes & 80 & 0.709 & $<0.0001$ \\
\hline Interstitial eosinophils & 80 & 0.377 & 0.024 \\
\hline \multicolumn{4}{|l|}{ Renal function injury } \\
\hline eGFR & 80 & -0.812 & $<0.0001$ \\
\hline Serum creatinine & 80 & 0.781 & $<0.0001$ \\
\hline Serum cystatin $\mathrm{C}$ & 76 & 0.844 & $<0.0001$ \\
\hline \multicolumn{4}{|l|}{ Tubular interstitial injury } \\
\hline Urine NAG & 79 & 0.563 & $<0.0001$ \\
\hline Urine lysozyme & 78 & 0.463 & $<0.001$ \\
\hline Urine RBP & 78 & 0.701 & $<0.0001$ \\
\hline RIV & 80 & 0.780 & $<0.0001$ \\
\hline \multicolumn{4}{|l|}{ Glomerular injury } \\
\hline Urine $\alpha 2$-microglobulin & 77 & 0.384 & 0.014 \\
\hline Urine C3 & 77 & 0.563 & $<0.0001$ \\
\hline $24 \mathrm{~h}$ urine protein & 80 & 0.729 & $<0.0001$ \\
\hline
\end{tabular}

Correlation analysis was carried out using Spearman coefficients with Bonferroni correction to adjust for multiple testing

diabetic nephropathy To explore whether MCs at different stages of diabetic nephropathy have different subtypes and functions, production of tryptase and chymase in the renal MCs was investigated. Immunohistochemical results showed that more than $80 \%$ of the tryptasepositive MCs in the sections of diabetic nephropathy patients and normal control participants were chymasepositive (Fig. 4). No obvious change was found in subtype of MCs in different groups of diabetic nephropathy patients.

\section{Expression changes of TGF- $\beta 1$, renin and $T N F-\alpha$ in renal} MCs during the development of diabetic nephropathy TGF$\beta 1$ and renin were detected in the MCs of normal control participants and diabetic nephropathy patients. No obvious change in these two molecules was found in the MCs of the diabetic nephropathy patients compared with the normal control participants (Fig. 5). Only very weak immunohistochemical staining for TNF- $\alpha$ was found in the MCs of normal controls; however, immunohistochemical staining for TNF- $\alpha$ increased in the MCs of diabetic nephropathy patients. The immunohistochemical staining intensity of $\mathrm{TNF}-\alpha$ increased gradually from the microalbuminuria stage to the proteinuria stage and renal insufficiency stage (Fig. 6).

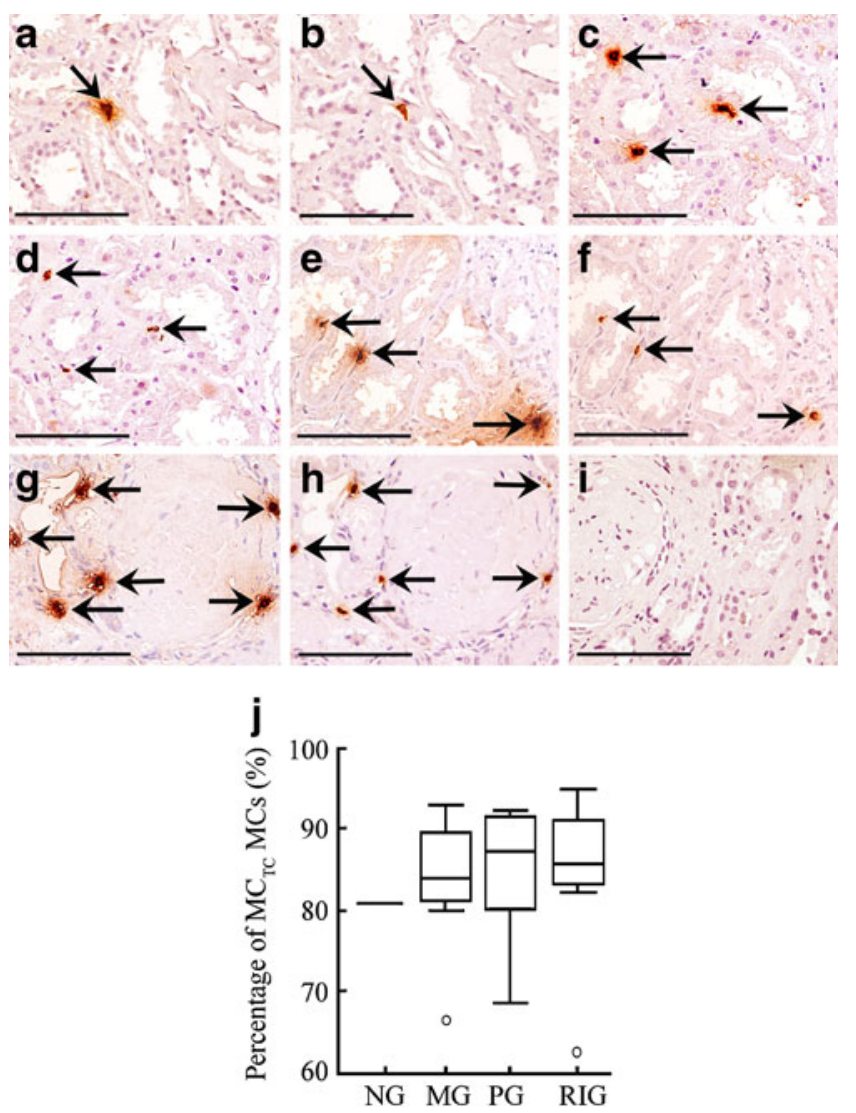

Fig. 4 Most of the tryptase-positive renal MCs (shown by black arrows) of normal controls and patients with diabetic nephropathy were found to be chymase-positive. Serial renal sections from 30 diabetic nephropathy patients (including microalbuminuria stage group $[n=10]$, proteinuria stage group $[n=10]$ and renal insufficiency stage group $[n=10])$ and ten normal controls were used in the assay. For the diabetic nephropathy patients, the percentage of $\mathrm{MC}_{\mathrm{TC}} \mathrm{MCs}$ was calculated for each section. As only a few MCs were found in each section from the normal controls, all the MCs found in all sections from the normal controls were pooled together and analysed. Multiple comparisons were performed using KruskalWallis ANOVA, and pairwise comparison was performed using Mann-Whitney $U$ test, with Bonferroni correction. a Normal control group, immunohistochemically stained for tryptase. b Normal control group, immunohistochemically stained for chymase. c Microalbuminuria stage group, immunohistochemically stained for tryptase. d Microalbuminuria stage group, immunohistochemically stained for chymase. e Proteinuria stage group, immunohistochemically stained for tryptase. f Proteinuria stage group, immunohistochemically stained for chymase. g Renal insufficiency stage group, immunohistochemically stained for tryptase. h Renal insufficiency stage group, immunohistochemically stained for chymase. i Negative control of immunohistochemistry. Scale bars (a-i) $100 \mu \mathrm{m}$. j Results of statistical analysis: the horizontal line represents the average ratio of $\mathrm{MC}_{\mathrm{TC}} \mathrm{MCs}$ in the normal control group. White circles represent extreme values where the extremes are defined as cases with values more than three box lengths from the upper or lower edge of the box. The box length is the interquartile range. a and $\mathbf{b}, \mathbf{c}$ and $\mathbf{d}, \mathbf{e}$ and $\mathbf{f}, \mathbf{g}$ and $\mathbf{h}$ are serial sections. MG, microalbuminuria stage group; NG, normal control group; PG, proteinuria stage group; RIG, renal insufficiency stage group 


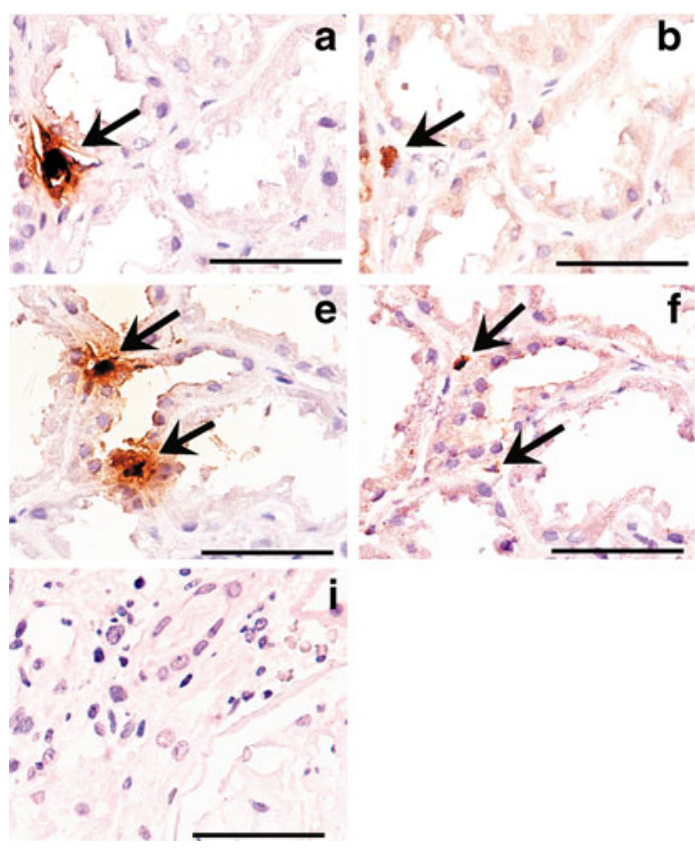

Fig. 5 TGF- $\beta 1$ and renin were detected in MCs (shown by black arrows) of normal controls and patients with diabetic nephropathy. No obvious change in these two molecules was found in the MCs of diabetic nephropathy patients compared with those of the normal controls. a Normal controls, immunohistochemically stained for tryptase. b Normal controls, immunohistochemically stained for renin. c Patients with diabetic nephropathy, immunohistochemically stained for tryptase.

\section{Discussion}

By using renal sections from diabetic nephropathy patients at different stages of disease, the present study investigated changes in MCs occurring during development of the disease and the association between renal MCs and the progression of diabetic nephropathy. Increased renal MCs were found at all stages of diabetic nephropathy and these paralleled the progression of the disease. As early as the microalbuminuria stage, MCs began to increase. As diabetic nephropathy progressed, renal MCs increased continually. Interestingly, in the present study, the degranulation level of MCs was also found to be increased with the development of diabetic nephropathy. MCs are rich in growth factors, proteases and inflammatory mediators. Once activated, MCs can release various kinds of pathogenic substances such as TGF- $\beta$, chymase, tryptase, histamine, renin and TNF- $\alpha$ by degranulation [2631]. Certainly, release of these kinds of substances can cause renal injury. Therefore, the increase in the number and degranulation level of MCs suggests that MCs could be involved in the development of diabetic nephropathy. In accordance with this, correlation analysis revealed that MCs correlated significantly with serum creatinine, serum cystatin $\mathrm{C}$ and the decline of GFR. d Patients with diabetic nephropathy, immunohistochemically stained for renin. e Normal controls, immunohistochemically stained for tryptase. f Normal controls, immunohistochemically stained for TGF- $\beta 1$. g Patients with diabetic nephropathy, immunohistochemically stained for tryptase. $\mathbf{h}$ Patients with diabetic nephropathy, immunohistochemically stained for TGF- $\beta 1$. i Negative control of immunohistochemistry. Scale bars $(\mathbf{a}-\mathbf{i}) 50 \mu \mathrm{m}$. $\mathbf{a}$ and $\mathbf{b}, \mathbf{c}$ and $\mathbf{d}, \mathbf{e}$ and $\mathbf{f}, \mathbf{g}$ and $\mathbf{h}$ are serial sections

Previous studies suggested that MCs contributed to renal diseases mainly through promotion of tubular interstitial injury [13, 15-17, 32-34]. Most investigations focused on the roles of MCs on renal fibrosis. Similar to previous studies [16], in the present study, MCs were found to be distributed mainly in the tubular interstitium, but rarely in the glomerulus. Furthermore, the present study showed that the increase in $\mathrm{MC}$ number and degranulation level occurred mainly at the later stages of diabetic nephropathy (in the proteinuria and renal insufficiency stage groups) when tubular interstitial injury became obvious. The involvement of MCs in tubular interstitial injury was also supported by the results of correlation analysis. MCs were found to be correlated significantly with urine RBP, urine lysozyme, urine NAG and RIV. In addition, renal MCs correlated significantly with the tubular interstitial inflammatory cells, including neutrophils, eosinophils, macrophages and $\mathrm{T}$ lymphocytes. The correlation between MCs and inflammatory cells suggests that MCs play a role in promoting renal inflammation in diabetic nephropathy.

MCs can be activated in several ways. The well-known, classical pathway of MC activation is through $\operatorname{IgE}-\mathrm{Fc} \varepsilon \mathrm{R}$ cross-linking. But MCs can also be activated through alternative pathways, such as the complement pathway and the microbial pattern recognition receptors (toll-like receptors) pathway. In vitro studies have demonstrated that $\mathrm{C} 3 \mathrm{a}$ is the 


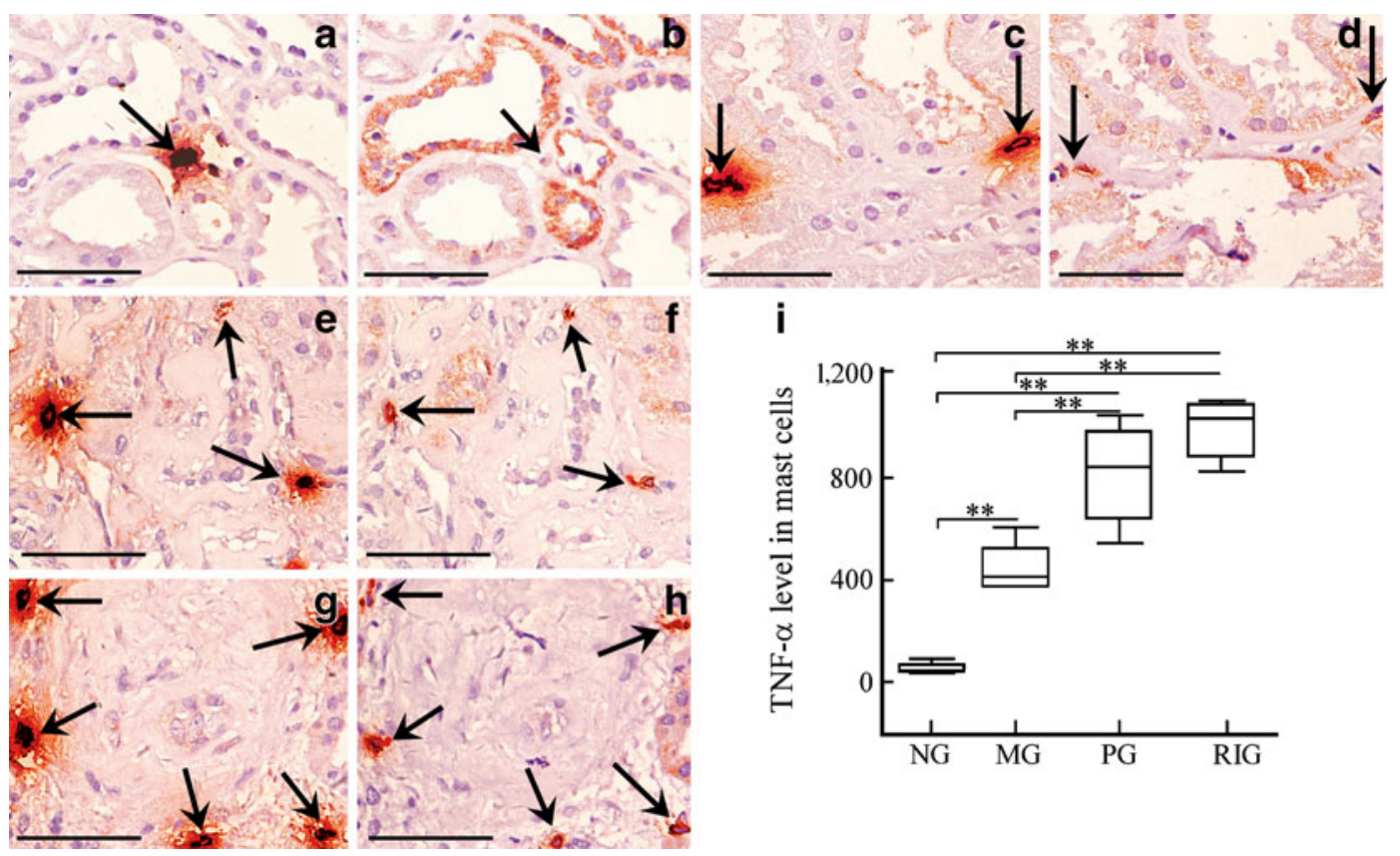

Fig. 6 TNF- $\alpha$ in renal MCs (shown by black arrows) was found to be increased with the development of diabetic nephropathy. Serial sections of renal biopsies from 30 patients with diabetic nephropathy (including microalbuminuria stage group $[n=10]$, proteinuria stage group $[n=10]$ and renal insufficiency stage group $[n=10])$ and ten normal controls were used in the assay. At least 15 randomly selected MCs were analysed in each section of diabetic nephropathy patients. As only a few MCs were found in each section from the normal controls, all the MCs found in all sections from the normal controls were pooled together and analysed (in total, $21 \mathrm{MCs}$ were found and analysed). Multiple comparisons were performed using Kruskal-Wallis ANOVA, and pairwise comparison was performed using MannWhitney $U$ test, with Bonferroni correction. a Normal control group,

most potent chemoattractant and activator of MCs [35, 36]. Activation of complement, which can lead to the generation of C3a, has also been demonstrated to increase in diabetic nephropathy [37]. Thus, we suggest that increased complement activation might contribute to the recruitment and activation of MCs.

Tryptase and chymase are two major proteinases in MCs. Studies have shown that these two enzymes play very important roles in the function of MCs [21]. According to the production of the two molecules, human MCs are classified into two subtypes [16]. The $\mathrm{MC}_{\mathrm{TC}} \mathrm{MCs}$ are believed to produce both tryptase and chymase, whereas the $\mathrm{MC}_{\mathrm{T}} \mathrm{MCs}$ are believed to produce tryptase only. The $\mathrm{MC}_{\mathrm{T}}$ $\mathrm{MCs}$ are believed to be $\mathrm{T}$ cell-dependent and the $\mathrm{MC}_{\mathrm{TC}}$ MCs are believed to be T cell-independent [38]. In human renal tissue, different results have been reported on $\mathrm{MC}$ subtype [11, 15, 17, 32-34]. MC subtype is believed to be disease-dependent [16]. In diabetic nephropathy, Rüger et al. reported that most MCs of diabetic nephropathy patients (about $80.3 \%$ ) contained both tryptase and chymase [11]. However, in another study, Okon and Stachura reported a immunohistochemically stained for tryptase. b Normal control group, immunohistochemically stained for TNF- $\alpha$. c Microalbuminuria stage group, immunohistochemically stained for tryptase. $\mathbf{d}$ Microalbuminuria stage group, immunohistochemically stained for TNF- $\alpha$. e Proteinuria stage group, immunohistochemically stained for tryptase. f Proteinuria stage group, immunohistochemically stained for TNF- $\alpha$. g Renal insufficiency stage group, immunohistochemically stained for tryptase. h Renal insufficiency stage group, immunohistochemically stained for TNF- $\alpha$. Scale bars $(\mathbf{a}-\mathbf{h}) 50 \mu \mathrm{m}$. i Results of quantitative analysis, ${ }^{* *} p<0.01$. $\mathbf{a}$ and $\mathbf{b}, \mathbf{c}$ and $\mathbf{d}, \mathbf{e}$ and $\mathbf{f}, \mathbf{g}$ and $\mathbf{h}$ are serial sections. MG, microalbuminuria stage group; $\mathrm{NG}$, normal control group; $\mathrm{PG}$, proteinuria stage group; RIG, renal insufficiency stage group

much lower ratio of chymase-positive MCs in diabetic nephropathy patients (about 52.9\%) [17]. In the present study, approximately $84 \%$ of the tryptase-positive MCs in diabetic nephropathy patients were found to be chymasepositive, and no obvious change in subtype was found in different groups of diabetic nephropathy patients. This result supports the idea that $\mathrm{MC}_{\mathrm{TC}}$ is the major subtype of renal MCs in diabetic nephropathy patients. Unexpectedly, in the present study, $80.5 \%$ of the MCs in normal control participants were found to be $\mathrm{MC}_{\mathrm{TC}}$ subtype. The result was quite different from what had been reported by Okon and Stachura [17] and Tóth et al. [33] (about 14.5\% and $20.1 \%$ of $\mathrm{MCs}$, respectively, in normal control participants were found to be $\mathrm{MC}_{\mathrm{TC}}$ subtype in their reports), but similar to the result of Rüger et al. [11] (about $62.5 \%$ of $\mathrm{MCs}$ in normal controls were found to be $\mathrm{MC}_{\mathrm{TC}}$ subtype in their report). Is $\mathrm{MC}_{\mathrm{TC}}$ the major subtype of renal MCs in healthy human beings? As only a few MCs could be found in each section of the normal control participants, investigations based on many more specimens are required. 
In the present study, we also investigated the expression of TGF- $\beta 1$, renin and TNF- $\alpha$ in MCs. As an important profibrotic factor, TGF- $\beta$ has been proven to play important roles in the pathogenesis of diabetic nephropathy [39-42]. As the key enzyme in the process of angiotensin production, renin participates in the regulation of extracellular volume, arterial vasoconstriction and blood pressure. Also, it has been reported that renin can upregulate TGF- $\beta$ expression $[43$, 44]. Recently, renin was reported to be able to induce renal inflammation through binding and activating prorenin receptors [45]. An increased renin level was reported in patients with diabetic nephropathy and treatment with renin inhibitor attenuated the development of the disease [46]. TNF- $\alpha$ is a pleiotropic cytokine. As an important proinflammatory molecule, TNF- $\alpha$ could promote the expression of adhesion molecules and the recruitment and activation of inflammatory cells $[47,48]$. In addition, TNF- $\alpha$ could increase production of reactive oxygen species [49]. For the first time, we demonstrated that TGF- $\beta$, renin and TNF- $\alpha$ are produced by human renal MCs. Interestingly, the level of TNF- $\alpha$ in MCs was found to be increased with the development of diabetic nephropathy. These findings further support the involvement of MCs in diabetic nephropathy, and also provide us with some clues for deeper understanding of the mechanisms through which MCs could exert their roles: (a) through synthesising and releasing TGF- $\beta$ and renin, MCs may contribute to tubular interstitial fibrosis; (b) through synthesising and releasing renin and TNF- $\alpha$, MCs may initiate and (or) promote tubular interstitial inflammation. These clues are helpful not only for understanding the mechanisms of diabetic nephropathy, but also for developing treatment strategies. For example, as a novel renin inhibitor, aliskiren may protect against diabetic nephropathy not only through blocking the renin-angiotensin system, but also through inhibiting MC degranulation-associated renal inflammation.

It should be pointed out that in the present study we could not identify the resident MCs and the infiltrated MCs. Thus, we could not differentiate their pathogenic roles in the development of diabetic nephropathy. MCs are highly heterogeneous. The microenvironment (for example the presence of different kinds of cytokines) in which the MCs reside determines the gene expression and phenotype development of the cells. With the variation of microenvironmental conditions, the phenotypes (for example, the expression level of TNF- $\alpha$, as shown in the present study) and pathogenic roles of MCs may differ to some degree during the development of diabetic nephropathy. The present study did not differentiate the pathogenic roles of MCs at microalbuminuria stage, macroalbuminuria stage and renal insufficiency stage. Also, the present study did not observe the influence of agents that antagonise the renin-angiotensin system on MC number. In a study based on a $5 / 6$ nephrectomy rat model, Jones et al. reported that
$\mathrm{ACE}$ inhibition led to a reduction in renal $\mathrm{MC}$ number and reduced renal injury [50]. As a possible new mechanism of renin-angiotensin system blockage therapy, it is interesting. Further exploration of these questions may provide helpful information for better understanding of the mechanisms of diabetic nephropathy and the pathogenic roles of MCs.

In summary, increased MCs and degranulation level were found at all stages of diabetic nephropathy and these paralleled the development of the disease. Increased MCs correlated significantly with the indices indicating tubular and interstitial injury. As MCs are multifunctional, the mechanisms of MC involvement in diabetic nephropathy are likely to be complex. However, as MCs have been proven to contain tryptase, chymase, TGF- $\beta$, renin and TNF- $\alpha$, theoretically they can contribute to diabetic nephropathy through synthesis and release of these molecules. Taken together, results from the present study suggest that MCs play important roles in the development of diabetic nephropathy, especially in tubular interstitial injury. However, further work is necessary to prove this.

Acknowledgements This study was supported by the Jingling Hospital Fund (2010 M027). We thank J. P. Wang and C. H. Zen, who are affiliated to the Research Institute of Nephrology, Jingling Hospital, for their help with histological analysis. We thank X. D. Zhu and M. C. Zhang, who are affiliated to the Research Institute of Nephrology, Jingling Hospital, for their help with immunohistochemical analysis. We also thank Y. X. Liu and M. L. Zhou, who are affiliated to the Data and Statistics Centre of Jingling Hospital for their help with data analysis.

Contribution statement JMZ conceived and designed the study, drafted the article, participated in the revision of the manuscript and gave final approval of the version to be published. GHY, ZC, RW and ZHL participated in the conception of the study, the analysis and interpretation of data and revising the article, and gave final approval of the version to be published.

Duality of interest The authors declare that there is no duality of interest associated with this manuscript.

\section{References}

1. Balakumar P, Arora MK, Ganti SS, Reddy J, Singh M (2009) Recent advances in pharmacotherapy for diabetic nephropathy: current perspectives and future directions. Pharmacol Res 60:24-32

2. Kawakami $T$ (2009) A crucial door to the mast cell mystery knocked in. J Immunol 18:6863-6881

3. Galli SJ, Kalesnikoff J, Grimbaldeston MA, Piliponsky AM, Williams CM, Tsai M (2005) Mast cells as "tunable" effector and immunoregulatory cells: recent advances. Annu Rev Immunol 23:749-786

4. Dawicki W, Marshall JS (2007) New and emerging roles for mast cells in host defence. Curr Opin Immunol 19:31-38

5. Sayed BA, Christy A, Quirion MR, Brown MA (2008) The master switch: the role of mast cells in autoimmunity and tolerance. Annu Rev Immunol 26:705-739 
6. Malaviya R, Abraham SN (2001) Mast cell modulation of immune responses to bacteria. Immunol Rev 179:16-24

7. Galli SJ, Nakae S, Tsai M (2005) Mast cells in the development of adaptive immune responses. Nat Immunol 6:135-142

8. Levick SP, McLarty JL, Murray DB, Freeman RM, Carver WE, Brower GL (2009) Cardiac mast cells mediate left ventricular fibrosis in the hypertensive rat heart. Hypertension 53: 1041-1047

9. Sakamoto-Ihara T, Suzuki Y, Kurusu A, Yamashita M, Horikoshi S, Tomino Y (2007) Possible involvement of mast cells in renal fibrosis in patients with IgA nephropathy. Inflamm Res 56:421-427

10. Melillo RM, Guarino V, Avilla E et al (2010) Mast cells have a protumorigenic role in human thyroid cancer. Oncogene 29:6203-6215

11. Rüger BM, Hasan Q, Greenhill NS, Davis PF, Dunbar PR, Neale TJ (1996) Mast cells and type VIII collagen in human diabetic nephropathy. Diabetologia 39:1215-1222

12. Hiromura K, Kurosawa M, Yano S, Naruse T (1998) Tubulointerstitial mast cell infiltration in glomerulonephritis. Am J Kidney Dis 32:593-599

13. Roberts IS, Brenchley PE (2000) Mast cells: the forgotten cells of renal fibrosis. J Clin Pathol 53:858-862

14. Kondo S, Kagami S, Kido H, Strutz F, Müller GA, Kuroda Y (2001) Role of mast cell tryptase in renal interstitial fibrosis. J Am Soc Nephrol 12:1668-1676

15. Goto E, Honjo S, Yamashita H, Shomori K, Adachi H, Ito H (2002) Mast cells in human allografted kidney: correlation with interstitial fibrosis. Clin Transplant 16(Suppl 8):7-11

16. Ehara T, Shigematsu H (2003) Mast cells in the kidney. Nephrology (Carlton) 8:130-138

17. Okon K, Stachura J (2007) Increased mast cell density in renal interstitium is correlated with relative interstitial volume, serum creatinine and urea especially in diabetic nephropathy but also in primary glomerulonephritis. Pol J Pathol 58:193-197

18. Holdsworth SR, Summers SA (2008) Role of mast cells in progressive renal diseases. J Am Soc Nephrol 19:2254-2261

19. Welker P, Krämer S, Groneberg DA et al (2008) Increased mast cell number in human hypertensive nephropathy. Am J Physiol Renal Physiol 295:F1103-F1109

20. Silva GE, Costa RS, Ravinal RC, dos Reis MA, Dantas M, Coimbra TM (2008) Mast cells, TGF-betal and alpha-SMA expression in IgA nephropathy. Dis Markers 24:181-190

21. Balakumar P, Reddy J, Singh M (2009) Do resident renal mast cells play a role in the pathogenesis of diabetic nephropathy? Mol Cell Biochem 330:187-192

22. Togawa H, Nakanishi K, Shima Y et al (2009) Increased chymasepositive mast cells in children with crescentic glomerulonephritis. Pediatr Nephrol 24:1071-1075

23. Tervaert TW, Mooyaart AL, Amann K et al (2010) Pathologic classification of diabetic nephropathy. J Am Soc Nephrol 21:556-563

24. Okon K, Szumera A, Kuzniewski M (2003) Are CD34+ cells found in renal interstitial fibrosis? Am J Nephrol 23:409-414

25. Singh LK, Boucher W, Pang X et al (1999) Potent mast cell degranulation and vascular permeability triggered by urocortin through activation of corticotropin-releasing hormone receptors. J Pharmacol Exp Ther 288:1349-1156

26. Metz M, Grimbaldeston MA, Nakae S, Piliponsky AM, Tsai M, Galli SJ (2007) Mast cells in the promotion and limitation of chronic inflammation. Immunol Rev 217:304-328

27. Nakae S, Suto H, Berry GJ, Galli SJ (2007) Mast cellderived TNF can promote Th17 cell-dependent neutrophil recruitment in ovalbumin-challenged OTII mice. Blood 109:3640-3648
28. Grimbaldeston MA, Nakae S, Kalesnikoff J, Tsai M, Galli SJ (2007) Mast cell-derived interleukin 10 limits skin pathology in contact dermatitis and chronic irradiation with ultraviolet B. Nat Immunol 8:1095-1104

29. Moon TC, St Laurent CD, Morris KE et al (2010) Advances in mast cell biology: new understanding of heterogeneity and function. Mucosal Immunol 3:111-128

30. Silver RB, Reid AC, Mackins CJ et al (2004) Mast cells: a unique source of renin. Proc Natl Acad Sci USA 101:1360713612

31. Helske S, Syväranta S, Kupari M et al (2006) Possible role for mast cell-derived cathepsin $\mathrm{G}$ in the adverse remodelling of stenotic aortic valves. Eur Heart J 27:1495-1504

32. Ehara T, Shigematsu H (1998) Contribution of mast cells to the tubulointerstitial lesions in IgA nephritis. Kidney Int $54: 1675-1683$

33. Tóth T, Tóth-Jakatics R, Jimi S, Takebayashi S (2000) Increased density of interstitial mast cells in amyloid A renal amyloidosis. Mod Pathol 13:1020-1028

34. Yamada M, Ueda M, Naruko T et al (2001) Mast cell chymase expression and mast cell phenotypes in human rejected kidneys. Kidney Int 59:1374-1381

35. Nilsson G, Johnell M, Hammer CH et al (1996) C3a and C5a are chemotaxins for human mast cells and act through distinct receptors via a pertussis toxin-sensitive signal transduction pathway. J Immunol 157:1693-1698

36. Hartmann K, Henz BM, Krüger-Krasagakes S et al (1997) C3a and $\mathrm{C} 5 \mathrm{a}$ stimulate chemotaxis of human mast cells. Blood 89:2863-2870

37. Hansen TK, Forsblom C, Saraheimo M et al (2010) Association between mannose-binding lectin, high-sensitivity C-reactive protein and the progression of diabetic nephropathy in type 1 diabetes. Diabetologia 53:1517-1524

38. Irani AM, Craig SS, DeBlois G, Elson CO, Schechter NM, Schwartz LB (1987) Deficiency of the tryptase positive, chymase negative mast cell type in gastrointestinal mucosa of patients with defective $\mathrm{T}$ lymphocyte function. J Immunol 138:4381-4386

39. Chen S, Jim B, Ziyadeh FN (2003) Diabetic nephropathy and transforming growth factor-beta: transforming our view of glomerulosclerosis and fibrosis build-up. Semin Nephrol 23:532-543

40. Border WA, Noble NA (1997) TGF-beta in kidney fibrosis: a target for gene therapy. Kidney Int 51:1388-1396

41. Border WA, Noble NA (1998) Evidence that TGF-beta should be a therapeutic target in diabetic nephropathy. Kidney Int 54:1390-1391

42. Sharma K, Jin Y, Guo J, Ziyadeh FN (1996) Neutralization of TGF-beta by anti-TGF-beta antibody attenuates kidney hypertrophy and the enhanced extracellular matrix gene expression in STZ-induced diabetic mice. Diabetes 45:522530

43. Huang Y, Noble NA, Zhang J, Xu C, Border WA (2007) Renin-stimulated TGF-betal expression is regulated by a mitogen-activated protein kinase in mesangial cells. Kidney Int $72: 45-52$

44. He M, Zhang L, Shao Y et al (2009) Inhibition of renin/ prorenin receptor attenuated mesangial cell proliferation and reduced associated fibrotic factor release. Eur J Pharmacol 606:155-161

45. Balakumar P, Jagadeesh G (2010) Cardiovascular and renal pathologic implications of prorenin, renin, and the (pro)renin receptor: promising young players from the old reninangiotensin-aldosterone system. J Cardiovasc Pharmacol 56:570-579 
46. Kelly DJ, Zhang Y, Moe G, Naik G, Gilbert RE (2007) Aliskiren, a novel renin inhibitor, is renoprotective in a model of advanced diabetic nephropathy in rats. Diabetologia 50:2398-2404

47. Holtmann MH, Neurath MF (2004) Differential TNF-signaling in chronic inflammatory disorders. Curr Mol Med 4:439-444

48. Matera MG, Calzetta L, Cazzola M (2010) TNF-alpha inhibitors in asthma and COPD: we must not throw the baby out with the bath water. Pulm Pharmacol Ther 23:121-128
49. Koike N, Takamura T, Kaneko S (2007) Induction of reactive oxygen species from isolated rat glomeruli by protein kinase $\mathrm{C}$ activation and TNF-alpha stimulation, and effects of a phosphodiesterase inhibitor. Life Sci 80:17211728

50. Jones SE, Kelly DJ, Cox AJ, Zhang Y, Gow RM, Gilbert RE (2003) Mast cell infiltration and chemokine expression in progressive renal disease. Kidney Int 64:906-913 